\title{
Design of an Assistive Walking Device with Special Rehabilitation Capabilities
}

\author{
Ahmad Muammar Bin Md Yasin, Lim Wei Liong, Patrick S. K. Chua ${ }^{*}$, Zheng Jianxin \\ Singapore Institute of Technology, Singapore
}

Copyright $\bigcirc 2016$ by authors, all rights reserved. Authors agree that this article remains permanently open access under the terms of the Creative Commons Attribution License 4.0 International License

\begin{abstract}
This paper presents a new novel design of a smart walker for rehabilitation purpose by patients in hospitals and rehabilitation centers. The design features a full frame walker that provides secured and stable support while being foldable and compact. It also has smart features such as telecommunication and patient activity monitoring.
\end{abstract}

Keywords Walker, Rehabilitation, Gait, Balance, Physiotherapist, Hospital

\section{Introduction}

The rapid ageing population in Singapore has resulted in an increase in healthcare problems associated with the growing number of elderly persons. A concern faced by the elderly is accidents involving a fall. It has been estimated that $17.2 \%$ of elderly Singaporeans above the age of 65 experiences falls [1]. Although most of them do not suffer from any injuries, $10 \%$ of the falls lead to severe injuries and even death. Studies have shown that the most frequent cause of accidents resulting in a fall is environment related [2]. The second cause is attributed to gait and balance disorders or weakness of the elderly person [2]. The existence of other medical problems such as heart diseases and stroke can cause weaknesses in limbs which can affect the ability of the affected person to balance properly [3].

Therapy should be employed once a person is diagnosed with a gait and movement balance disorder. The use of walking devices (walkers) by patients with gait and movement balance disorder helps in their ability to walk and balance properly. In 2012, Friedman [4] noted that "walking devices should be forcefully encouraged, especially to patients reluctant to use them either for vanity's sake, or because of fear of becoming dependent on them". Products such as rollators shown in Figure 1 and walking frames provide users with a fair level of support and balance.

Walking frames used in rehabilitation centers are usually large and cumbersome. Arm swing is also prohibited and flexion of the back is increased when holding on to the frame $[6,7]$. The low handles of a rollator results in unnecessary stress on the lower back.

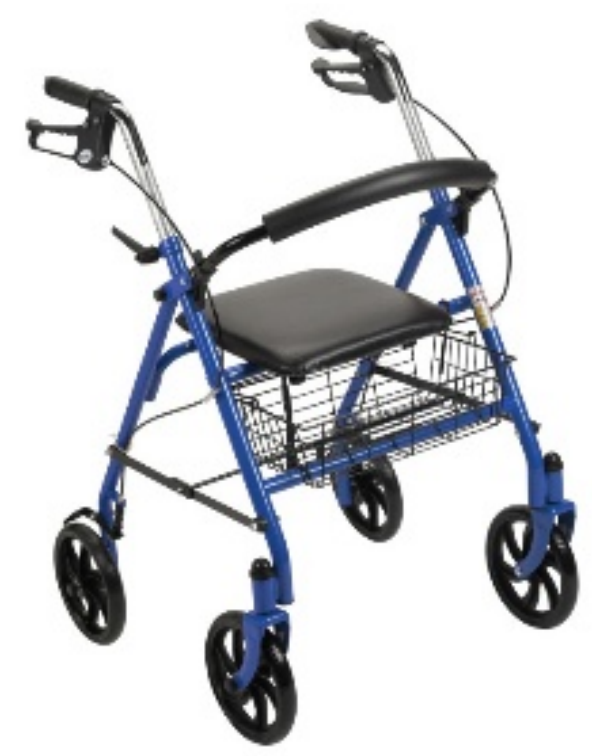

Figure 1. Rollator [5]

During a typical physiotherapy session, one therapist is usually required to accompany a patient so as to supervise and monitor the patient's progress. With the shortage of physiotherapists, the patients will have to be queued for the next available therapy session.

To resolve this problem, the efficiency and productivity of the physiotherapist has to be improved in terms of the number of patients a physiotherapist can handle within a therapy session. With the help of technology, telecommunication and other features can be integrated into the walker to make it smarter so that the physiotherapist need not accompany a patient during a therapy session all the time. Once the patient is doing the therapy correctly the physiotherapist can return to the rehabilitation station from which he/she can communicate with the patient and monitor the patient's rehabilitation activity remotely. 


\section{Objective}

The aim of this project is to design, fabricate and test a new unique walker that provides greater stability for the user as well as features that include telecommunication and rehabilitation activity monitoring.

\section{Existing Designs}

\subsection{Walkers}

A standard walker shown in Figure 2 is a four-point frame that stands approximately at waist height and surrounds the user on three sides. The standard walker has four legs with each leg ending in a rubber tip to prevent slippage during use. Wheels can be purchased separately and inserted to replace the rubber tips when required. Walkers are stable and are recommended for users with adequate back and arm strength to lift the walker and move it forward.

\subsection{Rollators}

Rollators are wheeled walking aids that comprise of a frame and three or four large wheels. The main difference between a walker and a rollator shown in Figure 2 is that a walker is simply a handled frame with legs.
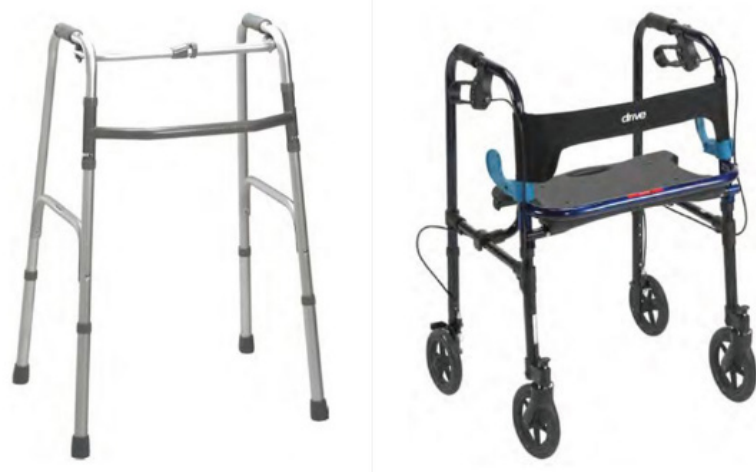

Figure 2. Drive medical walker and rollator [5]

A person with limited arm strength who has difficulty lifting should use rollator. Someone who is firm and stable will benefit from the maneuverability of a rollator compared to a walker. Rollators characteristically include an integrated flip-down or fixed seat for the user to rest on.

\subsection{Gait Trainers}

Gait trainers are designed for individuals who do not have the functional gait skills. Gait trainers shown in Figure 3 improve walking ability for children and adults with physical disabilities. A gait trainer offers postural alignment for gait practice. It provides more assistance in terms of balance and weight-bearing than a traditional rollator or walker. It also provides opportunities for the user to stand as it can bear weight in a safe and supported position.

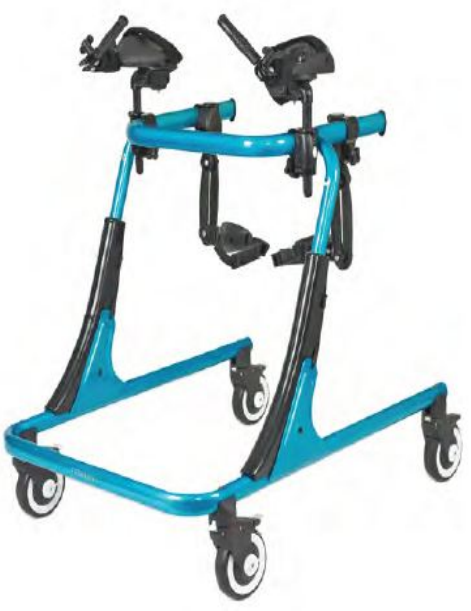

Figure 3. Gait trainer [5]

\subsection{Problems with Existing Products}

Fixed (non-wheeled) walker needs to be raised for each step therefore causing problems for patients with strength issues. The rollator (four-wheeled walkers) usually have foldable seat so that the user can rest when tired. However, rollators cause pain on the shoulders because most of the weight is supported by the arms [5]. Arm swing is also prohibited and flexion of the back is increased when holding on to the frame [5, 6]. Rollators, which are too light, can also cause stability issues. Gait trainers are mainly prescribed to patients with severe conditions such as stroke. As it is bulky and non-foldable, it can cause maneuverability issues. There is also a significant reduction in stride length when using the gait trainer.

\subsection{Walkie Version 1.0 Prototype}

A prototype walker named Walkie 1.0, shown in Figure 4 was developed and tested at a local private hospital and a charity health organization with elderly members. Its height is adjustable to suit the user. A bicycle seat allows the user to sit and rest when tired as well as to walk while sitting. A click -on/off safety harness is also provided to go round the patient's chest to secure the patient.

Many useful feedbacks were received some of which are as follows:

- Bicycle seat is uncomfortable and interferes with the users gait when walking.

- Walker needs to be foldable for storage to save space.

- $\quad$ Arm rest needed.

- $\quad$ Smart features should be incorporated e.g. telecommunication and patient rehabilitation activity monitoring 


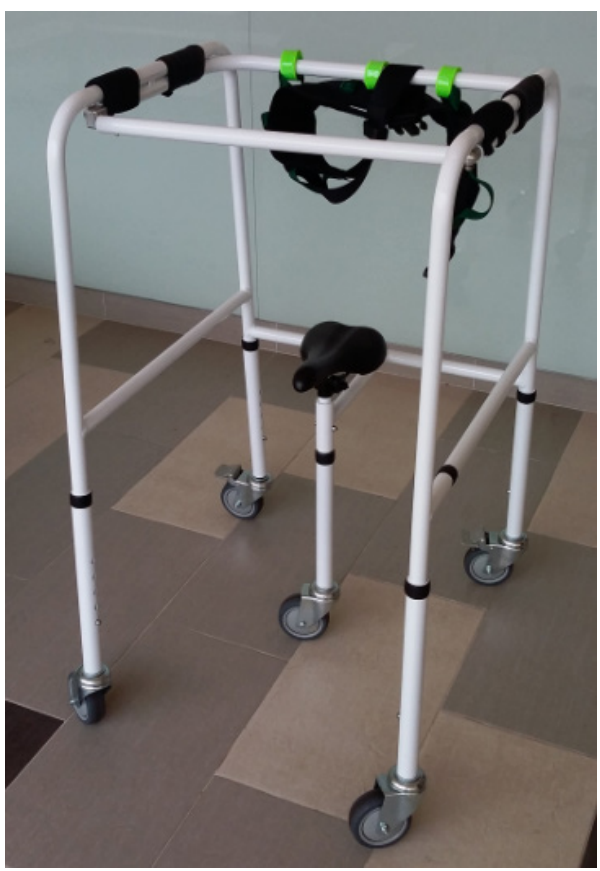

Figure 4. Walkie version 1.0 full-scale working prototype

\section{The New Walker Design}

A new design (Walkie version 2.0) was developed which incorporated the feedbacks received. The new design shown in Figure 5 aims to provide users with good support while being foldable and compact. It also possesses smart features for distance sensing, telecommunication and user rehabilitation activity monitoring.

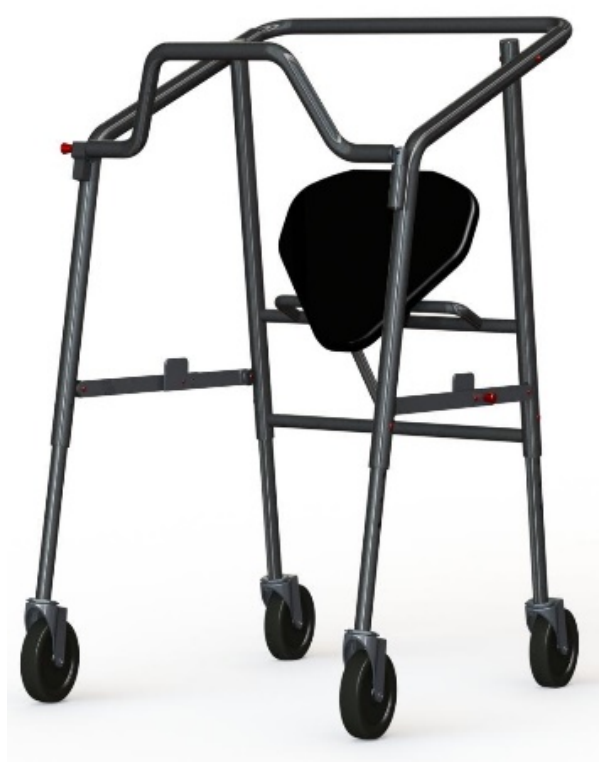

Figure 5. The smart walker.

\subsection{Folding Mechanism}

The new walker is foldable so as to save space during storage. The folding mechanism shown in Figure 6 features two retractable spring plungers that enable the folding mechanism to be locked and unlocked securely. The spring plunger shown in Figure 7 only requires a small amount of pulling force to retract.

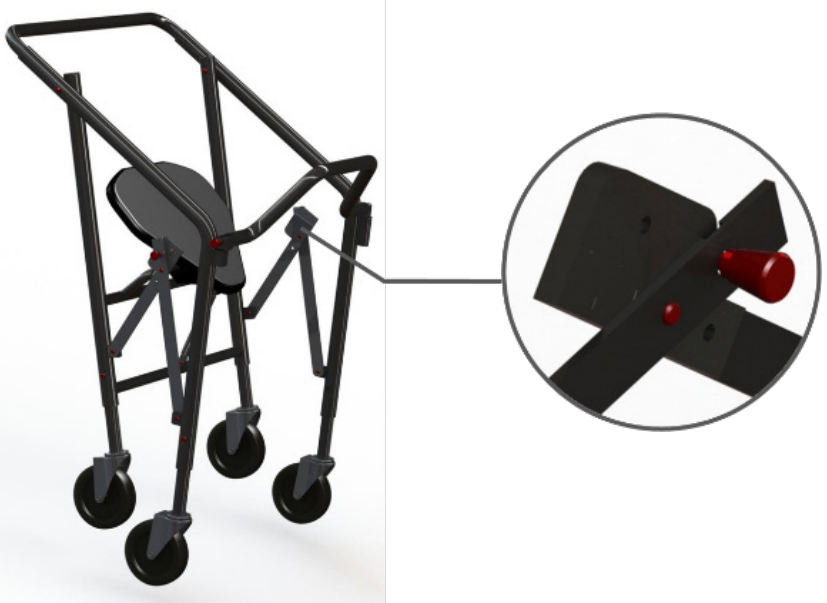

Figure 6. Spring plunger used for the folding mechanism.

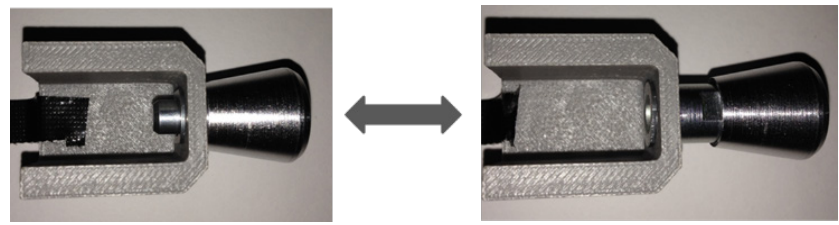

Figure 7. Retracted and extended spring plunger.

\subsection{Retractable Seat}

The seat features a tension gas spring that allows it to be folded automatically when the user applies a sitting force. The seat will then retract back to its original position when the user stands up as shown in Figure 8.

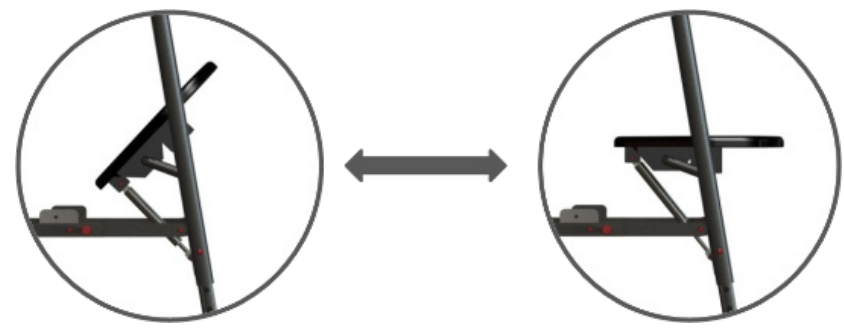

Figure 8. Retractable seat does not interfere with the user's gait.

\section{Electronics and Telecommunication}

In order to make the walker a smart device, several electronics have to be integrated into the walker. It allows the controller to perform the necessary processing. Sensors are 
incorporated to measure parameters such as distance walked, walking speed, patient's resting time, patient's mode of walking (sit-walking or stand-up walking) and duration, and it also allows voice communication between the physiotherapist and patient when the physiotherapist is at the physiotherapist base station.

\subsection{Controller}

Arduino UNO micro-controller with 14 digital input/output (IO) pins and 6 analog inputs was used as the controller. However, it is slower compared to Raspberry Pi but it can perform basic tasks like receiving and processing data. Cost-wise, Arduino UNO is also cheaper than Raspberry Pi 2 and using Raspberry Pi 2 as the controller for this system might be too overkill.

\subsection{Sensors}

In order to monitor the progress of the patient, sensors have to be used to retrieve information and gather the data for processing. There are several sensors and devices required to achieve the goal of measuring distance walked by the patient per therapy session, of knowing how long the patient has rested or doing the therapy at a sitting down position and if the patient has fallen down.

Distance - Hall Effect sensor which can measure the magnitude of the magnetic field at close proximity is used to detect distance covered by the walker during a therapy session. It is relatively small and operates electrically. As there is no physical moving part in the sensor it has a longer life cycle compared to that of the rotary encoder. The distance travelled can be measured by incorporating a magnet into the wheel of the walker. As the patient walks, the Hall Effect sensor will pick up the magnetic field at every wheel rotation. Each detection represents one wheel rotation and thus the distance walked can be calculated from the circumference of the wheel.

Fall Detection - An accelerometer is used to measure the rate of change in velocity. It can also be used to measure the angle of tilt in $\mathrm{X}$ and $\mathrm{Y}$ direction. When the accelerometer is tilted above the preset threshold, the therapist will be notified so that he/she can approach the patient to provide assistance.

Detect Sitting - in the case when the patient does not have enough strength to stand, he can continue the therapy by sitting down. This reduces the body load of the legs and at the same time he can still exercise the leg muscles by pushing himself along with the walker. A force resistive sensor is used to measure the amount of force exerted on the resistive surface. When the strip is stretched, it reduces the resistance and sends a signal to the controller. It is embedded under the seat cover with minimal footprint. Since it is flat, the user will not be able to feel it. The force resistive sensor does not have any mechanical part, thus giving it a longer life span compared to using a micro-switch. The downside of using the force sensor is the cost as it is comparably more expensive than the micro-switch.

\subsection{Wireless Transmission}

Since the patient using the walker must have the freedom to maneuver during the therapy session, the telecommunication device cannot have any hard wires connecting to the main controller where the therapist uses to monitor the patient's progress. Therefore, the information of the patient's progress has to be transmitted wirelessly. There are many wireless personal area networks (WPANs) technologies such as Wi-Fi, Bluetooth and ZigBee. ZigBee is a low-cost, low-power and wireless mesh network standard and it is intended to be simpler and less expensive than Wi-Fi and Bluetooth. XBee's ZigBee provides a cost-effective wireless connectivity using ZigBee technology. It has an indoor range of up to $30 \mathrm{~m}$ and outdoor line of sight range up to $90 \mathrm{~m}$ transmitting at $1 \mathrm{~mW}$. It can have up to 11 digital IO pins and 4 analog input pins. XBee Series 2 has a mesh routable network topology where it can be configured to be a coordinator, router or end device. In a mesh network there can only have 1 XBee coordinator where it is the main device to receive information. The XBee coordinator can be connected to multiple XBee routers and XBee end devices where the XBee routers are used as intermediate points to route information within the network so that the overall coverage can be extended from XBee end device to XBee coordinator.

\subsection{Voice Communication}

With this telecommunication device, the therapist does not need to be by the side of each patient. Thus a voice communication system has to be set up to allow the therapist and the patient to talk to each other in case of emergency or any urgent matters. This is especially good where the care center is huge and the patient may be far away from the therapist's desk. A simple existing technology can be used in this application to establish a voice communication such as using a set of walkie-talkies.

Walkie-talkie uses a half-duplex communication where at any one time, only one device is transmitting and another receiving and vice-versa. The remote walkie-talkie at the walker is taken apart to bring the necessary interfaces and feedback to the patient's hand support area such as the push-to-talk (PTT) button, microphone and speaker so that it will be easier for the patient to use. An emergency button is installed near the hand support area for the patient to press in case of emergency. When the patient presses the emergency button, his/her walkie-talkie channel will appear at the therapist's main controller's liquid crystal display (LCD) so that the physiotherapist can adjust his/ her walkie-talkie channel to talk to the patient.

\subsection{Privacy}

To protect the patient's privacy and interest, the monitoring and data logging system must only be used by authorized personnel and only after the patient has agreed to 
have such session. As it might be sensitive to some patients who are uncomfortable that their progress can be monitored by anyone, password protection has to be set up before any transmission could take place. After the authentication of the password, the system will begin transmitting the information to the physiotherapist's base computer for monitoring and logging.

\subsection{Software}

The main controller comes with Arduino IDE software which is used to create or edit the programing script for the controller. The proper script has to be uploaded to the controller before it can perform what it is tasked to do. In conjunction with the XBee shield and XBee, the software can add its supporting library to the program so that it can retrieve the data via the XBee medium and perform necessary processes.

To collect sensor values, the sensors and switches have to be connected to the XBee end device so that it can be transmitted to the XBee coordinator at the controller. The XBee end device has several pins that can be configured to be digital IO pins or analog pins. The configuration can be changed through XBee X-CTU software. This software can also change the operation of the XBee to coordinator, router or end device.

In order to monitor the patient's progress over time to determine if the patient's health condition is improving or deteriorating, the information for each therapy session of the patient will have to be logged. As the Arduino IDE does not have a logging system, other software could be used to perform such task such as Coolterm. Coolterm is a software that can record incoming information that was printed on the Arduino IDE serial monitor. This software is able to save the logs into text file. However, to analyze the data clearer, the text file can be exported to excel sheet using delimiters to sort the information so that it will be clearer for the physiotherapist to see.

\subsection{Future Enhancement}

To further improve the capability of the telecommunication device, indoor location positioning can be looked into in the future. With this added feature the physiotherapist can know where the patient is located at real time so that in any case of emergency, the physiotherapist can locate the patient quickly and effectively. This feature is especially good for a large care center with many patients undergoing physiotherapy simultaneously where finding the patient's location might be harder.

Another future enhancement that can be looked into is to establish a real working system in monitoring multiple XBee end devices since the XBee series 2 which uses mesh topology can communicate with multiple XBee end devices or routers.

\section{Design Validation}

Simulation was done using SolidWorks simulation to validate the structural integrity of the design.

\subsection{Static Stress Test and Weld Size}

Stress simulation was done using SolidWorks simulation. A load of $150 \mathrm{~kg}$ was applied on the seat of a simplified model as shown in Figure 9. The maximum stress, located on the weld joint is determined to be $99.6 \mathrm{MPa}$.

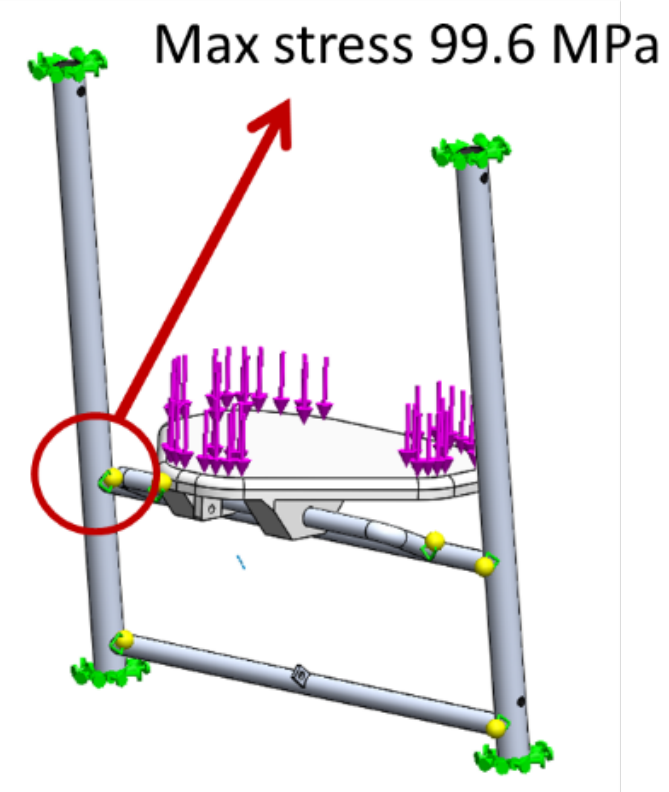

Figure 9. Simplified model for SolidWorks simulation.

It is essential to determine the weld sizes for the weld joints. SolidWorks can determine the minimum weld size using the same stress simulations and conditions as shown in Figure 10. Table 1 show that a minimum of $2.7 \mathrm{~mm}$ weld size is required to prevent failure.

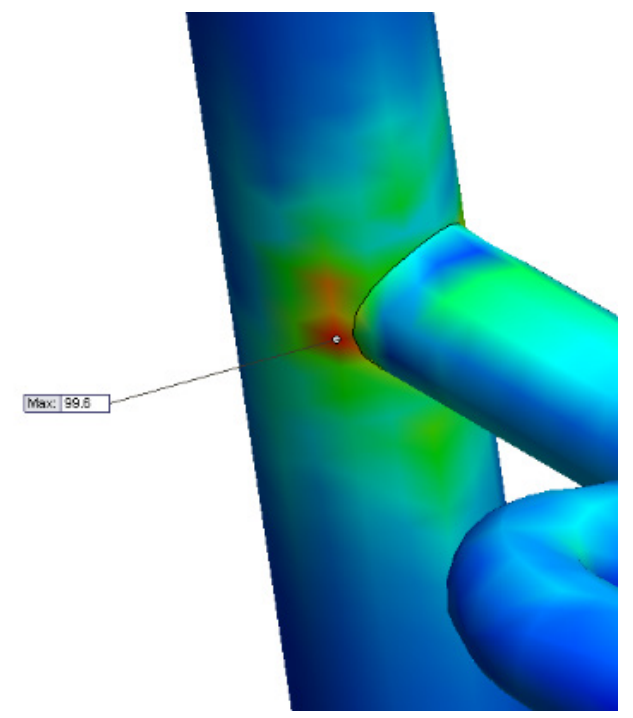

Figure 10. Maximum stress results from SolidWorks simulation. 
Table 1. Weld Size Simulation Results

\begin{tabular}{|c|c|c|c|}
\hline Type & Min & Max & Mean \\
\hline Weld size $(\mathrm{mm})$ & 0.75289 & 2.7287 & 1.8243 \\
\hline Weld throat size $(\mathrm{mm})$ & 0.75289 & 2.7287 & 1.8243 \\
\hline Joint normal force $(\mathrm{N})$ & $-1.4162 \mathrm{E}+005$ & $1.2877 \mathrm{E}+005$ & -1702.1 \\
\hline
\end{tabular}

\subsection{Minimum Pin Diameter}

Several pivoting pins are added to the new design to ensure the walker is foldable. The location of the pins is shown in Figure 11. The most common failure for this type of joint is shearing. Therefore it is important that the minimum diameters of the pins are calculated in order to prevent failure.

It is difficult to calculate the reaction forces exerted on the pins by hand when a load is applied on the seat. The forces however can be determined by using SolidWorks simulation. Figure 11 also shows the maximum shear forces for the $\mathrm{x}, \mathrm{y}$ and $\mathrm{z}$ component acting on the pins on location A. The pins experience the highest forces in the simulation and therefore their minimum diameter has to be determined.

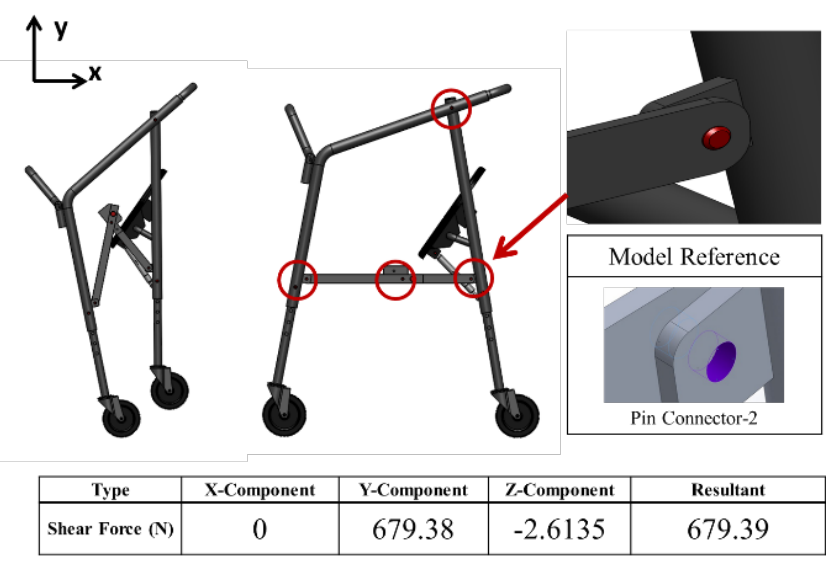

Figure 11. Shear force results for pivot pins.

Because the diameter of all pins on the new walker is the same, the maximum force shear force $679.38 \mathrm{~N}$ is used for the calculation. For single pin shear, the shear stress is found using Equation 1.

$$
\tau=F / A
$$

where $\tau$ is the shear stress, $F$ is the shear force and $A$ is the cross-sectional area.

For a force of $679.38 \mathrm{~N}$ (maximum shear force from simulation) and using AISI 1045 steel as the material, the yield strength $\tau_{y}$ is $450 M P a$.

Assuming safety factor of 5 , the allowable shear stress $\tau_{\text {allowable }}$ is $90 M P a$. The area of the pin can be calculated using

$$
A=F / \tau_{\text {allowable }}
$$

Therefore, the minimum diameter $d$ can be found as $3.1 \mathrm{~mm}$.

\section{Conclusions}

In this paper, the problems associated with existing mobility devices were discussed and the detailed presentation of the new smart walker design was presented. The use of smart features such as distance sensing, rehabilitation activity monitoring and telecommunication as well as the hardware involved were also explained. This smart walker is the first of its kind to be developed and is in line with the development of today's smart ward in a new hospital setting.

\section{Acknowledgement}

The authors would like to thank Singapore Institute of Technology and Kwong Wai Shiu Hospital for funding and supporting this project.

\section{REFERENCES}

[1] S. C. Lim, "Elderly Fallers: What Do We Need To Do?," Proceedings of Singapore Healthcare, pp. 154-158, 2010.

[2] L. Z. Rubenstein, "Falls in Older People: Epidemiology, Risk Factors and Strategies for Prevention," Age and Ageing, pp. ii37-ii41, 2006.

[3] H. E. Chin, "Losing One's Balance," Mind your body, p. 14, 20 March 2014.

[4] J. H. Friedman, "Gait Disorders in the Elderly," Medicine \& Health, pp. 84-85, March 2012.

[5] Drive Medical, "Drive Medical Product Catalog," 1 January 2014. [Online]. Available:

http://cdn.drivemedical.com/media/description/sections/MA STER_CAT_2014_VLR.pdf. [Accessed 1 October 2015].

[6] J. Crosbie, "Kinematics of Walking Frame Ambulation," Clinical Biomechanics, no. 8, pp. 31-36, 1993.

[7] D. K. Teodiano Freire Bastos-Filho, Devices for Mobility and Manipulation for People with Reduced Abilities, CRC Press, 2014. 